\title{
Phonological processing and arithmetic fact retrieval: Evidence from developmental dyslexia
}

\author{
Bert De Smedt ${ }^{*}, 1$, Bart Boets ${ }^{1}$ \\ Centre for Parenting, Child Welfare E Disabilities, Faculty of Psychology and Educational Sciences, Katholieke Universiteit Leuven, Belgium
}

\section{A R T I C L E I N F O}

\section{Article history:}

Received 9 December 2009

Received in revised form 11 October 2010

Accepted 12 October 2010

Available online 20 October 2010

\section{Keywords:}

Dyslexia

Fact retrieval

Arithmetic

Phonological processing

Angular gyrus

\begin{abstract}
A B S T R A C T
The triple-code model, cognitive neuroimaging and developmental behavioral data suggest a specific association between phonological processing and arithmetic fact retrieval. Accordingly, individuals with deficits in phonological processing, such as individuals with developmental dyslexia, are expected to show difficulties in arithmetic fact retrieval. The present study tested this proposal in 25 adults with developmental dyslexia and 25 matched controls by examining strategy use during single-digit multiplication and subtraction and its associations with phonological processing. Findings revealed that individuals with dyslexia retrieved fewer arithmetic facts from memory and were less efficient in doing so. At the same time, they showed deficits in phonological processing. Phonological processing, particularly phonological awareness, was related to arithmetic fact retrieval. This association was especially prominent in multiplication, indicating that fact retrieval in multiplication rather than subtraction is mediated by phonological processes. These data provide ground for future neuroimaging studies, who should examine the neural overlap between phonological processing and multiplication fact retrieval in the same sample of participants.
\end{abstract}

(C) 2010 Elsevier Ltd. All rights reserved.

\section{Introduction}

There are several reasons for suggesting a specific relation between phonological processing and arithmetic fact retrieval. The triple-code model postulates that numerals can be represented in a verbal-phonological code, which is used in verbally mediated arithmetic tasks such as retrieving arithmetic facts from memory, particularly in multiplication (Dehaene, 1992; Dehaene \& Cohen, 1995; Dehaene, Piazza, Pinel, \& Cohen, 2003). Cognitive neuroimaging data suggest a neural overlap between phonological processing and arithmetic fact retrieval in the left-temporo-parietal junction, in particular in the left angular and supramarginal gyri (Dehaene et al., 2003; Grabner et al., 2009; Pugh et al., 2001; Schlaggar \& Mccandliss, 2007; Vigneau et al., 2006). Recent developmental research shows a specific relation between phonological awareness and arithmetic fact retrieval (De Smedt, Taylor, Archibald, \& Ansari, 2010). If a reliable relation between phonological processing and arithmetic fact retrieval exists, then individuals with deficits

\footnotetext{
* Corresponding author at: Centre for Parenting, Child Welfare \& Disabilities, Katholieke Universiteit Leuven, Vesaliusstraat 2, Box 3765, Leuven, Belgium. Tel.: +32 163257 05; fax: +32 16325933 .

E-mail addresses: Bert.DeSmedt@ped.kuleuven.be, bert.desmedt@ped.kuleuven.ac.be (B. De Smedt).

1 Both authors equally contributed to this study and should be regarded as joint first authors.
}

in phonological processing, such as individuals with developmental dyslexia (e.g., Ramus et al., 2003; Snowling, 2000; Vellutino, Fletcher, Snowling, \& Scanlon, 2004), are expected to show difficulties with arithmetic fact retrieval. Although such a relation has been postulated theoretically (Dehaene et al., 2003; Simmons \& Singleton, 2008), there are, to our knowledge, no empirical studies that have investigated this association systematically. The present study therefore aimed to examine this association in adults with developmental dyslexia, a neurodevelopmental disorder in learning to read and to write despite normal IQ and adequate instruction. Such data might further shed light on the general associations between phonological processing and arithmetic fact retrieval and their underlying neural correlates.

\subsection{Arithmetic in dyslexia}

Several studies have indicated that multiplication is difficult for children with dyslexia (Miles, 1983; Simmons \& Singleton, 2008; Turner Ellis, Miles, \& Wheeler, 1996), although these studies did not systematically examine other operations or the strategies that children apply during problem solving. Landerl, Bevan, and Butterworth (2004) investigated single-digit arithmetic in children with dyscalculia, children with dyslexia and children with comorbid dyscalculia and dyslexia. These authors did not find significant group differences between children with dyslexia without dyscalculia and controls on single-digit addition, subtraction and multiplication. However, a careful inspection of their reaction time 
data suggests that children with dyslexia were slower than controls in multiplication and addition, but not in subtraction. It is also unclear whether the children identified as dyslexic in this study had phonological impairments. Thus, the precise arithmetical difficulties of children with dyslexia remain to be determined.

Studies on arithmetic in adults with developmental dyslexia are scarce. Temple (1991) reported a 19-year-old female patient with developmental phonological dyslexia, without manifest neurological impairment, who showed a specific deficit in multiplication. This patient had no other difficulties in calculation and her number processing skills were normal.

Simmons and Singleton (2006) provided the first systematic investigation of different single-digit arithmetic operations, comprising addition, subtraction and multiplication, in university students with developmental dyslexia. They showed that these students were less accurate in subtraction and multiplication. Students with dyslexia were slower in addition and subtraction, but there was only a trend for group differences in multiplication. More recently, Göbel and Snowling (2010) demonstrated that adults with developmental dyslexia, who had normal number processing skills, were significantly slower than controls in single-digit addition and multiplication. No group differences in accuracy were observed, which contrasts with earlier findings by Simmons and Singleton (2006).

Against the background of their data, Simmons and Singleton (2006) and Göbel and Snowling (2010) concluded that individuals with dyslexia have difficulties in arithmetic fact retrieval. This conclusion should be interpreted with caution as the authors did not assess the strategies that participants applied during problem solving. Indeed, studies that collected verbal protocol data to examine single-digit arithmetic strategy use reported that adults use a variety of strategies (e.g., Campbell \& Xue, 2001; Grabner et al., 2009; Lefevre, Sadesky, \& Bisanz, 1996). Although adults may often retrieve the answer to a problem directly from memory, other strategies are also applied; for example, calculation of the answer by means of a procedure or by counting, both of which are more time-consuming and rely stronger on magnitude processing and working memory. Grabner et al. (2009) demonstrated that verbal reports of different strategies correlated with activity in different brain regions: retrieval use was related to activation in the left angular gyrus whereas procedure use engaged a wider fronto-parietal network. For the hypothesis of fact retrieval difficulties in dyslexia to be supported, it needs to be demonstrated that individuals with developmental dyslexia either report fewer fact retrieval use or that they perform less accurately or more slowly when retrieving arithmetic facts from memory. To date, no such strategy data exist.

\subsection{Arithmetic fact retrieval and phonological processing}

Simmons and Singleton (2008) suggested that the poor arithmetic fact retrieval in dyslexia might be explained by their weak phonological processing skills. An association between phonological processing and arithmetic has been observed in typically developing children (De Smedt et al., 2010; Fuchs et al., 2005; Hecht, Torgesen, Wagner, \& Rashotte, 2001). More specifically, De Smedt et al. (2010) showed that in third graders fact retrieval was uniquely associated with phonological awareness but not with phonological memory. These authors suggested that the quality of phonological representations may be a key factor for efficient fact retrieval in a way that more distinct phonological representations contribute to a more efficient, i.e., faster and more accurate, retrieval of arithmetic facts. Few studies have addressed this association between phonological processing and fact retrieval in healthy adults. For example, Lee and Kang (2002) revealed in a dual-task experiment that phonological memory load impaired performance in single-digit multiplication but not in subtraction. Others, however, failed to find an effect of phonological memory load on single-digit multiplication performance (De Rammelaere, Stuyven, \& Vandierendonck, 1999; Seitz \& Schumann Hengsteler, 2000). Similarly, Simmons and Singleton found no association between verbal short-term memory and arithmetic fact retrieval in dyslexia, but it should be noted that their sample size was very small. Taken together, behavioral findings on the association between phonological processing and arithmetic fact retrieval remain inconclusive.

Cognitive neuroimaging data might further shed light on this issue. Neuroimaging studies of arithmetic have revealed that particularly the left angular gyrus is engaged during arithmetic problem types that are solved by means of fact retrieval (Grabner et al., 2007; Grabner et al., 2009). It appears that these left temporoparietal areas are also activated during phonological decoding and during tasks that involve effortful phonological analysis (Gelfand \& Bookheimer, 2003; Pugh et al., 2001; Shaywitz et al., 1998), suggesting that those tasks that put the highest demands on phonological representations are expected to reveal the strongest associations with arithmetic fact retrieval. Furthermore, individuals with dyslexia show less activation in the left temporo-parietal junction during tasks that involve effortful phonological analysis (McCandliss \& Noble, 2003; Pugh et al., 2001; Shaywitz et al., 1998; Temple, 2002). Against this background, it can be hypothesized that the arithmetic fact retrieval difficulties in dyslexia are due to their phonological processing deficits.

\subsection{The present study}

The present study aimed to examine arithmetic fact retrieval and its relation with phonological processing in adults with developmental dyslexia and matched controls. We collected strategy assessments during single-digit multiplication and subtraction to directly test whether adults with developmental dyslexia have difficulties in retrieving arithmetic facts. We further investigated whether these fact retrieval difficulties could be explained by individual differences in the three classic areas of phonological processing, i.e., phonological awareness, lexical access to long-term memory, and verbal short-term memory (Wagner \& Torgesen, 1987).

We expected that participants with dyslexia would retrieve fewer arithmetic facts from memory than controls. We also hypothesized that participants with dyslexia would show poor performance on the administered phonological measures and we expected associations between phonological processing and arithmetic fact retrieval use. Because fact retrieval is more often used in multiplication than in subtraction (e.g., Campbell \& Xue, 2001) and because multiplication shows increased activation in the left angular gyrus compared to subtraction, suggesting that it relies more on a verbal code than subtraction (Chochon, Cohen, Van De Moortele, \& Dehaene, 1999; Dehaene et al., 2003; Lee, 2000), we expected that group differences in arithmetic would be most prominent in multiplication and we predicted that associations between phonological processing and arithmetic would be stronger for multiplication than for subtraction.

It is important to note that difficulties in arithmetic fact retrieval may be due to a deficit in representing numerical magnitudes, as is the case in developmental dyscalculia (Butterworth, 2005; Landerl et al., 2004; Wilson \& Dehaene, 2007). Individuals with this neurodevelopmental disorder have a core deficit in understanding and manipulating numerical magnitudes, due to abnormalities in the intraparietal sulcus, which lead to general deficits in arithmetic (Butterworth, 2005; Landerl et al., 2004; Wilson \& Dehaene, 2007). Most important, developmental dyscalculia and developmental dyslexia are known to co-occur frequently (e.g., Shalev, 2007). The arithmetic difficulties of this comorbid subgroup of individuals with developmental dyslexia might be explained by their impaired 
magnitude processing rather than by their phonological weaknesses (Landerl et al., 2004). When examining the relation between phonological processing and arithmetic fact retrieval in developmental dyslexia, it is thus crucial to focus on those individuals with developmental dyslexia without dyscalculia, hence without difficulties in understanding numerical magnitudes. Against this background, we only selected individuals with developmental dyslexia without a formal diagnosis of developmental dyscalculia.

Three control measures were additionally administered to rule out alternative explanations for group differences in arithmetic. It is widely established that individuals with dyslexia are slower in identifying familiar symbols, including digits (e.g., Bowers \& Ishaik, 2003; Willburger, Fussenegger, Moll, Wood, \& Landerl, 2008). This slower digit identification might already explain individual differences in reaction times on arithmetic tasks. To account for this effect, a speeded digit identification task was included.

Although we investigated people with dyslexia without a formal diagnosis of developmental dyscalculia, we wanted to rule out that these subjects had a deficit in understanding of numerical magnitudes. To evaluate this possibility, a nonsymbolic magnitude comparison task was administered.

Because motor difficulties have been observed in dyslexia (e.g., Nicolson \& Fawcett, 1994; Ramus, 2003), we also administered a simple motor choice reaction time task. This allowed us to control for effects of general motor speed on the magnitude comparison task.

\section{Methods}

\subsection{Participants}

Participants were 25 university students ( 9 men, 16 women; aged $18-28$ years) with dyslexia and 25 healthy normal reading controls ( 8 men, 17 women; aged 18-29 years) recruited from the Katholieke Universiteit Leuven, Belgium. All participants with dyslexia received a formal diagnosis of developmental dyslexia. None of them received a formal diagnosis of developmental dyscalculia. None of them showed a history of psychiatric morbidity. In line with current practice in Belgium and the Netherlands (e.g., Gersons-Wolfensberger \& Ruijssenaars, 1997), they all performed below the 10th percentile compared to typically developing 16 -year-old readers on a Dutch standardized test for reading achievement (van den Bos et al., 1994). Control participants were university students individually matched in terms of study discipline and age to the students with developmental dyslexia. All controls had normal reading development and none of them had received a formal diagnosis of developmental dyscalculia. They all performed above the 50th percentile on the same standardized reading test (van den Bos et al., 1994) compared to typically developing 16 -year-old readers. Table 1 shows the descriptive statistics of both groups. There were no differences in age $(t(48)=0.22, p=.82, d=0.06)$. All participants had a normal non-verbal IQ (WAIS-III Matrix Reasoning) and no group differences on this measure were observed $(t(48)=0.32, p=.75, d=0.09)$. The groups differed significantly on the administered standardized reading test, i.e., word reading $(t(48)=-10.40, p<.01, d=-3.00)$ and nonword reading $(t(48)=-14.29, p<.01$, $d=-4.13)$.

\subsection{Procedure}

All participants were individually tested in a quiet room at our department during two sessions. The first test session involved the assessment of the arithmetic and control tasks, and took approximately $1 \mathrm{~h}$. The phonological measures were administered 2 months later in a second test session, which took approximately $30 \mathrm{~min}$.

\section{Table 1}

Descriptive statistics of the sample.

\begin{tabular}{lrrrrr}
\hline Variable & \multicolumn{2}{c}{ Dyslexia } & & \multicolumn{2}{c}{ Controls } \\
\cline { 2 - 3 } \cline { 5 - 6 } & \multicolumn{1}{c}{$M$} & & \multicolumn{1}{c}{$S D$} & & \multicolumn{1}{c}{$M$} \\
\hline Age (years) & 21.08 & 2.37 & & 20.93 & 2.09 \\
Non-verbal IQ & 107.88 & 13.24 & & 106.92 & 7.38 \\
Word reading $^{\mathrm{a}}$ & 65.72 & 10.13 & & 96.28 & 10.64 \\
Nonword reading $^{\mathrm{b}}$ & 55.28 & 9.32 & & 103.00 & 13.85 \\
\hline
\end{tabular}

a Number of correctly read items within $1 \mathrm{~min}$.

b Number of correctly read items within 2 min.

\subsection{Measures}

\subsubsection{Arithmetic}

The arithmetic tasks were presented with the E-prime 1.0 software (Schneider, Eschmann, \& Zuccolotto, 2002) on a 17-in. desktop computer. Stimuli occurred in white on a black background in Arial font (size 72). The experimenter initiated each trial by means of a control key. Each trial started with a 250 ms fixation in the centre of the computer screen. After $1000 \mathrm{~ms}$ the stimulus appeared and remained on screen until response. Responses were verbal. Timing started when the stimulus appeared and ended when the verbal response triggered the sound-activated voice-key. Participants were instructed to perform both accurately and quickly.

A multiplication task and a subtraction task were administered. The problems for these tasks were selected from all possible pairwise combinations of the numbers between 2 and 9 with the exclusion of tie problems (e.g., $2 \times 2$ ) and problems containing a 0 or 1 as operand or answer, which yielded 56 problems per operation. For multiplication, we selected one of each possible commutative pair, thereby counterbalancing the position of the largest number, which resulted in 28 trials. The subtraction problems were formed by reversing the 56 possible single-digit addition problems. Of this set, 28 problems were randomly selected. In half of them, the subtrahend was larger than the difference $(12-8)$; the other half contained problems with the difference larger than the subtrahend $(13-4)$.

All problems were presented in a horizontal format ( $14-6=)$. Participants could use whatever strategy they wanted to. Strategy use was recorded on a trial-bytrial basis by asking participants immediately after each response to describe how they got the answer. Such verbal reports are a reliable and valid way of assessing strategy use in arithmetic (e.g., Campbell \& Xue, 2001; Grabner et al., 2009; Lefevre et al., 1996). Similar to other studies on strategy use in single-digit arithmetic (e.g., Campbell \& Xue, 2001; Grabner et al., 2009; Lefevre et al., 1996), strategies were classified into retrieval (the participant responds immediately and there is no evidence of overt calculations) and procedure (the participant calculates the answer by decomposing the problem into smaller facts or by a counting procedure). The other category was added to include “don't know" answers and ambiguous responses (e.g., "I guessed the answer").

\subsubsection{Phonological processing}

2.3.2.1. Phonological awareness. In transparent orthographies, such as German and Dutch, phonological awareness tasks are known to reach ceiling levels in accuracy in primary school children, even in those with dyslexia (Landerl \& Wimmer, 2000; van Daal \& van der Leij, 1999). Therefore, the present study considered reaction time (of the correct trials) as the dependent variable in the phonological awareness tasks. A phoneme deletion task and a spoonerism task were administered. Both tasks were presented auditorily with the E-prime 1.0 software (Schneider et al., 2002). Timing started to run after the stimulus was presented and ended when the response of the participant was terminated. This was carried out by the experimenter who pressed the spacebar key as soon as the utterance of the participant was terminated.

In the phoneme deletion task, the participant had to delete a particular phoneme from a nonword (e.g., NORF without $/ \mathrm{r} /$ ). The task consisted of three blocks that each contained eight items and were preceded by two practice items. The first block involved the deletion of the second phoneme of a one-syllable nonword with onset cluster. The second block required the deletion of the penultimate phoneme of a one-syllable nonword with offset cluster. In the last block, the middle phoneme of a two-syllable nonword had to be deleted.

In the spoonerism task, the participant had to swap the initial phonemes of two presented words (e.g. LIME - HORSE) in order to create two new nonwords (HIME - LORSE). The task consisted of three blocks of eight items, with each block being preceded by two practice items. The first block involved two words with a single consonant onset. The second block comprised two words with a consonant cluster onset. In the last block, two nonwords with a single consonant onset were presented.

2.3.2.2. Lexical access. Lexical access was evaluated with three classic serial rapid naming tasks, which involved the naming of highly familiar objects, colors and letters (van den Bos, Zijlstra, \& Spelberg, 2002). Each task involved the presentation of a card of 50 stimuli randomly arranged in 5 columns of 10 stimuli, with each stimulus appearing 10 times. The participant was instructed to serially name the stimuli on the card as quickly and as accurately as possible. The time to complete the card was recorded for each task. Object naming involved the naming of five high-frequent, one-syllable words: boom ('tree'), eend ('duck'), stoel ('chair'), schaar ('scissors') and fiets ('bicycle'). Color naming comprised the colors black, blue, red, yellow and green, which were represented by small rectangles. Letter naming involved the naming of the lowercase letters $\mathrm{d}, \mathrm{o}, \mathrm{a}, \mathrm{s}$ and $\mathrm{p}$.

2.3.2.3. Verbal short-term memory. A digit span task and nonword repetition task were administered to measure verbal short-term memory. Digit span involved the immediate serial recall of spoken lists of digits between one and nine. Stimuli were 24 sequences of items with a list length increasing from 2 to 9 digits, with 3 stimuli for each list length. The sequences were presented auditorily via a CD recording of a professional speech therapist. The score on the test comprised the number of correctly recalled sequences.

A test of nonword repetition is frequently used as a pure measure of verbal short-term memory because the nonwords used in this test involve unfamiliar 
phonological sequences, which limits the use of long-term memory representations to support recall (Gathercole, Willis, Baddeley, \& Emslie, 1994). The Dutch adaptation (Scheltinga, 2003) of the Children's Test of Nonword Repetition (Gathercole et al., 1994) was used. Only the more difficult items of this test were administered. This test consisted of 36 nonwords that ranged from three to five syllables, with 12 trials for each syllable length. The nonwords, pronounced at a consistent rate, were recorded on $\mathrm{CD}$ by a professional speech therapist and this recording was presented. The participant had to repeat the nonword immediately after its presentation. The score on the test comprised the number of correctly recalled nonwords.

\subsubsection{Control tasks}

2.3.3.1. Speed of digit identification. This task was presented with the E-prime 1.0 software (Schneider et al., 2002) on a 17-in. desktop computer. Stimuli involved each of the digits 0-9, which were successively presented in white on a black background in Arial font (size 72). Each digit was presented twice, which yielded 20 trials. The experimenter initiated each trial by means of a control key. A trial started with a $250 \mathrm{~ms}$ fixation in the centre of the computer screen; after $1000 \mathrm{~ms}$ the stimulus appeared and remained on screen until response. Participants were instructed to name each digit as fast as possible. Timing started when the stimulus appeared and ended when the verbal response triggered the sound-activated voice-key.

2.3.3.2. Nonsymbolic magnitude comparison. This task was also presented with the E-prime 1.0 software (Schneider et al., 2002) on a 17-in. desktop computer. Participants had to indicate the larger of two simultaneously displayed arrays of dots one displayed on the left and one displayed on the right on the computer screen. The position of the largest numerosity was counterbalanced. Stimuli comprised all combinations of the numerosities $1-9$, which resulted in 72 trials. The stimuli were generated by means of the MATLAB script provided by Piazza, Izard, Pinel, Le Bihan, \& Dehaene (2004) and were controlled for non-numerical parameters, i.e., individual dot size, total occupied area, and density. This ensures that participants could not reliably use these non-numerical cues or perceptual features to make a correct decision. Each trial was initiated by the experimenter with a control key. A trial started with a 250 ms fixation; after $1000 \mathrm{~ms}$ the stimulus appeared and remained on screen until response. Participants were asked to respond by pressing a key on the keyboard corresponding to the side on which the largest numerosity was presented. The left response key was the ' $d$ ' key; the right response key was the ' $k$ ' key. Both keys were marked with blue stickers. Task instructions stressed both accuracy and speed.

2.3.3.3. Motor choice reaction time. The task design and administration was the same as in the numerical magnitude comparison task. Two shapes, one of which was filled, were displayed simultaneously - one displayed on the left, one displayed on the right. Participants had to press the key corresponding to the side on which the filled shape was presented. All shapes were similar in size. The administered shapes were circle, triangle, square, star and heart. Each shape occurred four times filled and four times non-filled, which resulted in 20 trials. The position of the filled shape was counterbalanced.

\section{Results}

Trials with voice-key failures (261/3952 or 6.60\%) were excluded from further analyses. Reaction time data and strategy frequency data were calculated for the correct trials only. Trials with more than $3 S D$ from a participants' mean reaction time were additionally excluded. Cohen's $d$ and partial eta-squared were computed as measures of effect size. The control tasks are discussed first. After that, the arithmetic and phonological processing data are described. Finally, we report the associations between phonological processing and arithmetic fact retrieval.

\subsection{Control tasks}

\subsubsection{Speed of digit identification}

Accuracy was very high and above $99 \%$ in both groups. Participants with developmental dyslexia $(M=542 \mathrm{~ms} ; S D=75)$ were significantly slower in identifying digits than controls $(M=467 \mathrm{~ms}$; $S D=33 ; t(48)=4.59, p<.01, d=1.32$ ). Therefore, the speed of digit identification was included as a covariate in subsequent analyses of the arithmetic tasks that considered reaction times.

\subsubsection{Motor choice reaction time}

Both groups performed with high accuracy (>99\%). Participants with dyslexia $(M=355 \mathrm{~ms} ; S D=38)$ were slower than controls $(M=333 \mathrm{~ms} ; S D=45)$ and this difference approached conventional levels of statistical significance $(t(48)=1.89, p=.07, d=0.54)$. This was taken into account when analyzing the reaction time data on the nonsymbolic magnitude comparison task.

\subsubsection{Nonsymbolic magnitude comparison}

Accuracy was high and at ceiling in participants with dyslexia $(M=98 \%)$ and in controls $(M=97 \%)$. Whenever people have to compare two numerical magnitudes, a distance effect occurs: when the numerical difference or distance between the two numerosities is small, people are slower and less accurate in deciding which of two numerosities is the larger than when the distance is large (Moyer \& Landauer, 1967). The size of this distance effect can be used as an indicator of an individual's understanding of numerical magnitude (Holloway \& Ansari, 2009). Therefore, reaction times were analyzed by calculating for each individual a linear regression in which the difference or distance between the displayed numerosities predicted reaction time. The slope of this regression line reflects the well-known effect of distance on participants' reaction times. This slope should be negative, because the distance effect predicts a negative relation between distance and reaction time. The intercept of this regression line reflects the general reaction time needed to compare both numerosities. The mean slope was negative in participants with dyslexia $(M=-35 \mathrm{~ms} ; S D=13)$ and in controls $(M=-32 \mathrm{~ms} ; S D=13)$. A significant distance effect was found in both groups as the slope differed significantly from 0 in participants with dyslexia $(t(24)=-13.72, p<.01, d=-2.53)$ and in controls $(t(24)=-12.65, p<.01, d=-2.74)$. There was no significant difference in the slope between participants with dyslexia and controls $(t(48)=0.69, p=.49, d=0.20)$, indicating that the distance effect was the same in both groups. The mean intercept was higher in participants with dyslexia $(M=669 \mathrm{~ms} ; S D=102)$ than in controls $(M=611 \mathrm{~ms} ; S D=100)$. This difference was small but it approached conventional levels of statistical significance $(F(1$, $\left.48)=4.03, p=.05, \eta_{p}^{2}=.08\right)$. However, this difference entirely disappeared when performance on the motor choice reaction time task was taken into account $\left(F(1,47)=1.41, p=.24, \eta_{p}^{2}=.03\right)$. In summary, these data indicate that the participants with dyslexia had no significant deficits in understanding numerical magnitudes.

\subsection{Arithmetic}

The mean error rates and reaction times for multiplication and subtraction are shown in Fig. 1. A 2 (operations) $\times 2$ (groups) repeated measures ANOVA with operation as a within-subject factor and group as a between-subjects factor was conducted on the error rates and reaction times. With regard to error rate, there was

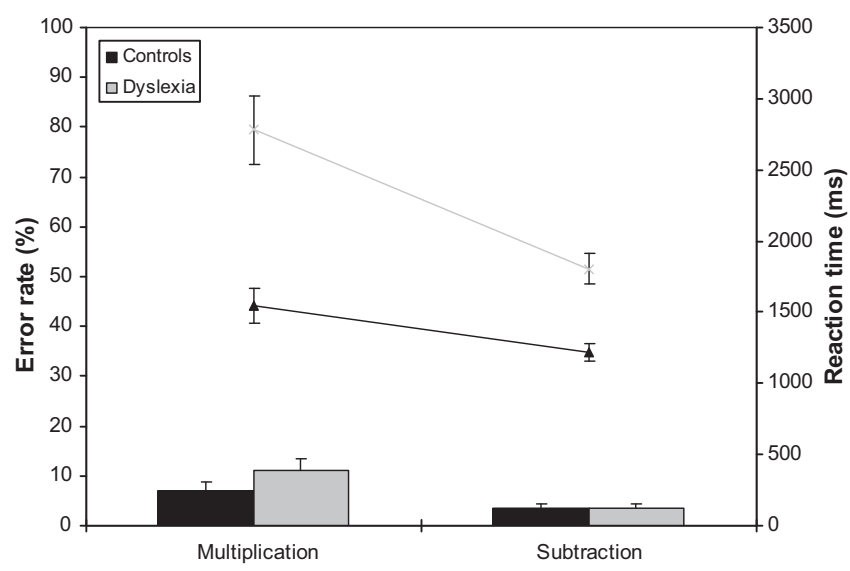

Fig. 1. Mean error rate and reaction time by operation on the arithmetic task. Bars depict error rates on the left $y$-axis and lines represent reaction times on the right $y$-axis. Error bars depict $1 S E$ of the mean. 
Table 2

Mean number of correct trials on which retrieval and procedures were used for each operation and group.

\begin{tabular}{|c|c|c|c|c|}
\hline \multirow[t]{2}{*}{ Task } & \multicolumn{2}{|c|}{ Dyslexia } & \multicolumn{2}{|c|}{ Controls } \\
\hline & $M$ & $S D$ & $M$ & $S D$ \\
\hline \multicolumn{5}{|l|}{ Multiplication } \\
\hline Retrieval & 13.56 & 5.24 & 18.68 & 4.23 \\
\hline Procedure & 8.40 & 4.60 & 4.48 & 3.40 \\
\hline \multicolumn{5}{|l|}{ Subtraction } \\
\hline Retrieval & 12.76 & 5.51 & 15.62 & 5.13 \\
\hline Procedure & 12.24 & 5.10 & 9.29 & 5.37 \\
\hline
\end{tabular}

a main effect of operation $\left(F(1,48)=20.06, p<.01, \eta_{p}^{2}=.29\right)$. There was no main effect of group $\left(F(1,48)=1.70, p=.20, \eta_{p}^{2}=.03\right)$ and no Group $\times$ Operation interaction $\left(F(1,48)=2.59, p=.11, \eta_{p}^{2}=.05\right)$.

The analysis of the reaction times showed a main effect of operation $\left(F(1,48)=42.88, p<.01, \eta_{p}^{2}=.47\right)$ with subtraction being solved faster than multiplication. There was a main effect of group $\left(F(1,48)=24.31, p<.01, \eta_{p}{ }^{2}=.34\right)$, indicating that individuals with dyslexia were slower than controls. There was also a significant Group $\times$ Operation interaction $\left(F(1,48)=10.55, p<.01, \eta_{p}^{2}=.18\right)$, suggesting that the difference between multiplication and subtraction was larger in participants with dyslexia than in controls. Group differences remained when the speed of digit identification was additionally controlled for $\left(F(1,47)=9.46, p<.01, \eta_{p}{ }^{2}=.17\right)$.

\subsubsection{Strategy use}

3.2.1.1. Strategy distribution. The strategy distribution was determined per operation by calculating for each participant the frequency with which a particular strategy was used on the correctly solved trials. Due to equipment failure, strategy data were not available for one control participant in subtraction.

The frequency of trials belonging to the other category was less than $1 \%$ of all trials. The mean frequencies of retrieval and procedural strategy use for each operation and group are shown in Table 2. A 2 (operations) $\times 2$ (groups) repeated measures ANOVA with operation as a within-subject factor and group as a betweensubjects factor was conducted on the frequencies of retrieval use. There was a main effect of operation $(F(1,47)=6.13, p=.02$, $\eta_{p}{ }^{2}=.12$ ), indicating more frequent retrieval use in multiplication than in subtraction. There was also a main effect of group $(F(1$, $47)=11.26, p<.01, \eta_{p}^{2}=.19$ ), with participants with dyslexia showing significantly less frequent retrieval use. The Operation $\times$ Group interaction was not significant $\left(F(1,47)=2.18, p=.15, \eta_{p}^{2}=.04\right)$, but group differences tended to be larger in multiplication than in subtraction. A similar 2 (operations) $\times 2$ (groups) repeated measures ANOVA was conducted on the frequencies of procedure use. These findings were, logically, the reverse of those observed for retrieval. Procedures were used more in subtraction than in multiplication $\left(F(1,47)=28.05, p<.01, \eta_{p}^{2}=.37\right)$ and participants with dyslexia showed higher frequencies of procedure use than controls $\left(F(1,47)=11.16, p<.01, \eta_{p}^{2}=.19\right)$. There was no Operation $\times$ Group interaction $\left(F(1,47)=0.45, p=.50, \eta_{p}^{2}=.01\right)$.

3.2.1.2. Strategy efficiency. We also examined the efficiency, in terms of error rate and reaction time, with which retrieval and procedural strategies were executed. Fig. 2 shows the mean error rate and reaction time for each strategy per operation per group. A 2 (operations) $\times 2$ (strategies) $\times 2$ (groups) repeated measures ANOVA with operation (multiplication vs. subtraction) and strategy (retrieval vs. procedure) as within-subject factors and group (dyslexia vs. controls) as a between-subjects factor was calculated on the error rates and reaction times. With regard to error rate, there were main effects of strategy $(F(1,43)=14.36, p<.01$, $\left.\eta_{p}{ }^{2}=.25\right)$ and operation $\left(F(1,43)=14.45, p<.01, \eta_{p}^{2}=.25\right)$ and a sig-

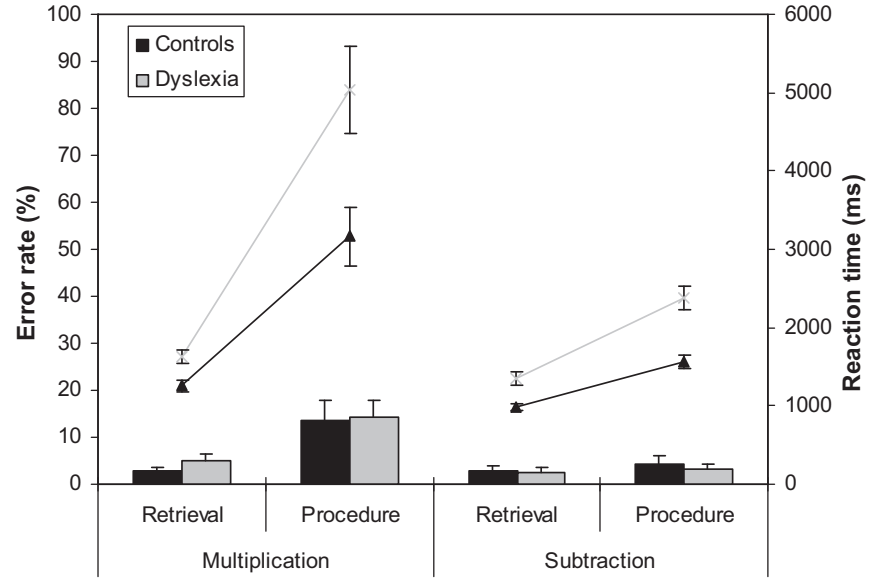

Fig. 2. Mean error rate and reaction time by strategy on the arithmetic task. Bars depict error rates on the left $y$-axis and lines represent reaction times on the right $y$-axis. Error bars depict $1 S E$ of the mean.

nificant Strategy $\times$ Operation interaction $(F(1,43)=11.16, p<.01$, $\left.\eta_{p}^{2}=.21\right)$. This interaction indicated that differences between retrieval and procedural strategies were only prominent in multiplication. There was no main effect of group $(F(1,43)=0.18, p=.67$, $\left.\eta_{p}{ }^{2}=.00\right)$ and group membership did not interact with operation $\left(F(1,43)=0.86, p=.36, \eta_{p}^{2}=.02\right)$, strategy $(F(1,43)=0.03, p=.81$, $\left.\eta_{p}{ }^{2}=.00\right)$ and the Strategy $\times$ Operation interaction $(F(1,43)=0.02$, $\left.p=.90, \eta_{p}^{2}=.00\right)$.

The analysis of the reaction times showed main effects of operation $\left(F(1,39)=46.05, p<.01, \eta_{p}^{2}=.54\right)$ and strategy $(F(1,39)=93.89$, $\left.p<.01, \eta_{p}^{2}=.71\right)$. Subtraction was executed faster than multiplication and retrieval strategies were completed more quickly than procedural strategies. There was a significant Strategy $\times$ Operation interaction $\left(F(1,39)=30.72, p<.01, \eta_{p}^{2}=.44\right)$, indicating that the reaction time differences between retrieval and procedural strategies were larger for multiplication than for subtraction. There was a main effect of group $\left(F(1,39)=11.63, p<.01, \eta_{p}^{2}=.23\right)$. Group interacted with strategy $\left(F(1,39)=9.16, p<.01, \eta_{p}^{2}=.19\right)$ and there was a trend towards a Group $\times$ Strategy $\times$ Operation interaction $(F(1$, $\left.39)=3.69, p=.06, \eta_{p}^{2}=.09\right)$. When the speed of digit identification was additionally controlled for only the main effect of group remained $\left(F(1,38)=4.56, p=.04, \eta_{p}^{2}=.11\right)$. In all, this indicates that participants with dyslexia were slower in executing both retrieval and procedural strategies.

\subsection{Phonological processing}

Table 3 shows the descriptive statistics on the administered phonological tasks. Findings indicate that participants with dyslexia performed more poorly than controls on each of these tasks. Prior to the correlational analyses, we aggregated performance on the phonological measures into composites along the suggested theoretical phonological components (Wagner \& Torgesen, 1987). Composite scores were created by calculating for each task $z$ scores on the total sample. The $z$ scores that were thought to measure the same phonological component were then averaged to create composite scores for phonological awareness (Phoneme deletion, Spoonerism), lexical access (Color naming, Object naming, Letter naming) and verbal short-term memory (Digit span, Nonword repetition). Because the measures of phonological awareness and lexical access were based on reaction time, the signs of the $z$ scores were reversed, to simplify interpretation. Thus, higher $z$ scores indicated better performance. Participants with dyslexia performed more poorly than controls on each of these phonological composites: Phonological awareness: $t(48)=-6.73$, 
Table 3

Descriptive statistics on the administered phonological tasks.

\begin{tabular}{|c|c|c|c|c|c|c|}
\hline \multirow[t]{2}{*}{ Task } & \multicolumn{2}{|l|}{ Dyslexia } & \multicolumn{2}{|l|}{ Controls } & \multirow[t]{2}{*}{$t$} & \multirow[t]{2}{*}{$d$} \\
\hline & $M$ & $S D$ & $M$ & $S D$ & & \\
\hline \multicolumn{7}{|l|}{ Phonological awareness } \\
\hline Phoneme deletion (ms) & 3309.49 & 1375.95 & 1448.49 & 643.83 & $6.13^{* *}$ & 1.77 \\
\hline Spoonerism (ms) & 7407.47 & 2824.63 & 4275.55 & 1262.01 & $5.06^{* *}$ & 1.46 \\
\hline \multicolumn{7}{|l|}{ Lexical access } \\
\hline Color naming (s) & 31.48 & 5.14 & 26.40 & 3.28 & $4.17^{* *}$ & 1.20 \\
\hline Object naming (s) & 35.28 & 5.55 & 29.88 & 2.70 & $4.38^{* *}$ & 1.26 \\
\hline Letter naming (s) & 24.20 & 5.71 & 17.08 & 2.64 & $5.66^{* *}$ & 1.63 \\
\hline \multicolumn{7}{|l|}{ Verbal short-term memory } \\
\hline Digit span & 11.56 & 1.87 & 14.40 & 2.50 & $-4.55^{* *}$ & -1.31 \\
\hline Nonword repetition & 21.24 & 4.47 & 26.04 & 4.41 & $-3.82^{* *}$ & -1.10 \\
\hline
\end{tabular}

${ }^{* *} p<.01$.

$p<.01, d=-1.94$; Lexical access: $t(48)=-5.99, p<.01, d=-1.73$; Verbal short-term memory: $t(48)=-5.48, p<.01, d=-1.58$.

\subsection{Correlations between phonological processing and arithmetic fact retrieval}

Pearson correlation coefficients were calculated to examine the associations between the phonological composites and arithmetic fact retrieval. These associations were investigated at the total group level and at the subgroup level for each operation separately. To obtain an unbiased measure of arithmetic fact retrieval ability, we used the participants' frequency of retrieval (i.e., the number of the correctly retrieved items) on the multiplication and the subtraction task. This measure was selected because indices of retrieval efficiency (i.e., accuracy and speed) might be less suited to examine correlations: the accuracy of retrieval was high, creating a problem of range restrictions; the speed of retrieval was based on significantly more trials in controls than in participants with dyslexia, and therefore such measure is biased because in participants with dyslexia it may contain only (relatively) easy retrieval items whereas in controls, it might comprise both easy and more difficult items.

\subsubsection{Total group level}

Overall correlations between phonological abilities and fact retrieval frequency are expected to occur simply because the two groups differ on both dimensions. If real associations between phonological abilities and fact retrieval exist, these associations should be observed when the defining group characteristic, reading ability, is additionally controlled for. We therefore calculated partial correlations between the phonological abilities and retrieval frequency, with the effect of reading ability, as measured by the word reading ability test, partialed out (Table 4 ). In multiplication, both phonological awareness and verbal short-term memory were significantly related to the frequency of fact retrieval. The relation between lexical access and multiplication fact retrieval was substantial but only tended to approach statistical significance $(r(50)=.28, p=.06)$. In subtraction, however, only a significant association between phonological awareness and fact retrieval emerged.
Table 4

Correlations between retrieval frequency and phonological measures controlled for individual differences in reading ability.

\begin{tabular}{llll}
\hline Task & $\begin{array}{l}\text { Phonological } \\
\text { awareness }\end{array}$ & Lexical access & $\begin{array}{l}\text { Verbal short-term } \\
\text { memory }\end{array}$ \\
\hline Multiplication & $.53^{* *}$ & .28 & $.35^{*}$ \\
Subtraction & $.30^{*}$ & .01 & .20 \\
\hline
\end{tabular}

${ }^{*} p<.05$.

** $p<.01$.

A hierarchical regression analysis was subsequently calculated to determine which of the administered phonological abilities contributed unique variance to multiplication fact retrieval. In Step 1 , reading ability was entered as a predictor. In Step 2, phonological awareness, lexical access and verbal short-term memory were simultaneously entered into the model. Findings revealed that only phonological awareness $(\beta=.56, t=2.81, p<.01$, Unique $\left.R^{2}=.11\right)$ but neither lexical access $(\beta=.24, t=1.42, p=.16$, Unique $\left.R^{2}=.03\right)$ nor verbal short-term memory $(\beta=.06, t=0.32, p=.75$, Unique $R^{2}=.00$ ) predicted unique variance in multiplication fact retrieval.

\subsubsection{Subgroup level}

The correlations between the phonological measures and retrieval frequency in multiplication and subtraction are displayed per subgroup in Table 5 . In control participants, there was a significant association between phonological awareness and multiplication fact retrieval (see also Fig. 3), whereas no other significant relations were observed. This association remained significant, even when individual differences in reading ability were controlled for $(r(25)=.43, p=.04)$.

Turning to the correlations in the participants with dyslexia, a significant association was observed between phonological awareness and retrieval frequency in multiplication. No other significant associations between phonological abilities and retrieval frequency were found. As shown in Fig. 3, the association between phonological awareness and multiplication fact retrieval was similar to the one observed in the control group. Likewise, this association remained significant when individual differences in reading ability were controlled for $(r(25)=.42, p=.04)$. Different from the control group, there was a trend for an association between phonologi-

Table 5

Correlations between retrieval frequency and phonological measures for the participants with dyslexia and controls.

\begin{tabular}{|c|c|c|c|c|c|c|}
\hline \multirow[t]{2}{*}{ Task } & \multicolumn{3}{|l|}{ Controls } & \multicolumn{3}{|l|}{ Dyslexia } \\
\hline & Phonological awareness & Lexical access & Verbal short-term memory & Phonological awareness & Lexical access & Verbal short-term memory \\
\hline Multiplication & $.40^{*}$ & .04 & .19 & $.41^{*}$ & .17 & .23 \\
\hline Subtraction & .06 & -.17 & .02 & .37 & .01 & .29 \\
\hline
\end{tabular}

${ }^{*} p<.05$. 

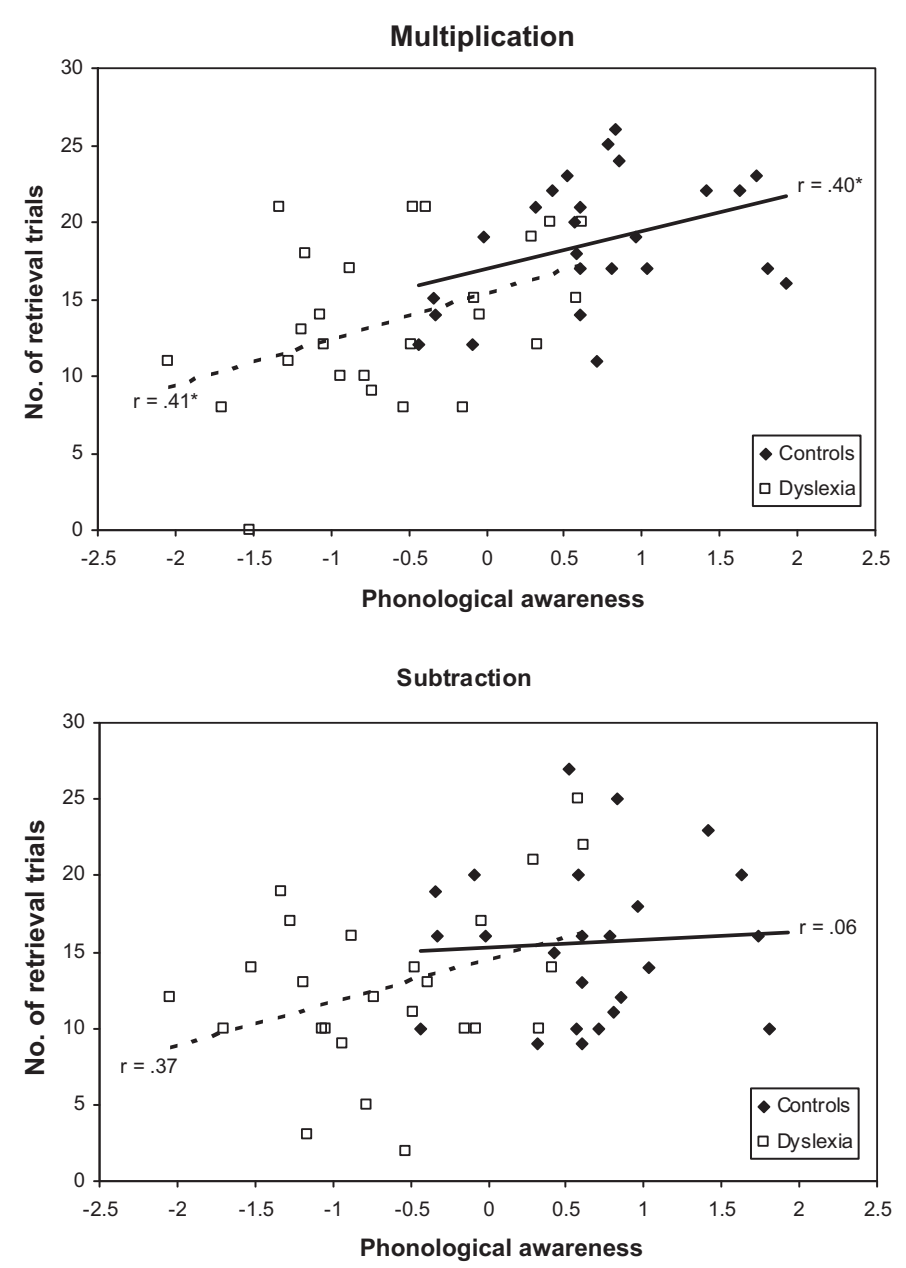

Fig. 3. Scatterplots showing the associations between phonological awareness and the frequency of retrieval in multiplication (top) and subtraction (bottom). The linear regressions for these associations are depicted by dashed lines for participants with dyslexia and by solid lines for the control group.

cal awareness and subtraction fact retrieval $(r(25)=.37, p=.07)$. A careful inspection of Fig. 3, however, indicated that this association was primarily driven by two individuals, who had very high frequencies of subtraction fact retrieval. When these participants were removed, the association between phonological awareness and subtraction fact retrieval entirely disappeared $(r(23)=.11, p=.61)$, which is in line with the findings from the control group.

\section{Discussion}

Do individuals with dyslexia have difficulties in arithmetic fact retrieval? Consistent with Simmons and Singleton (2006) and with Göbel and Snowling (2010), we showed that participants with dyslexia were significantly slower than matched controls on singledigit arithmetic, even when the speed of digit identification was controlled for. Consistent with Göbel and Snowling (2010), but different from Simmons and Singleton (2006), there were no group differences in accuracy. Extending these findings by means of strategy assessment data, we have shown for the first time that individuals with developmental dyslexia retrieve fewer facts from memory, as reflected in their significantly lowered frequencies of retrieval use. Fact retrieval was additionally less efficient in participants with dyslexia, as they were significantly slower than controls in executing retrieval strategies. In contrast to our expectations, the group differences in arithmetical performance were not more pro- nounced in multiplication than in subtraction. In all, these findings converge to the conclusion of fact retrieval difficulties in dyslexia.

The present study also revealed that participants with dyslexia were slower in executing not only retrieval but also procedural strategies. The latter might be explained by the weaker fact retrieval in dyslexia. The procedures used by adults in single-digit arithmetic typically comprise the decomposition of the problem into smaller facts (e.g., $14-8=14-4=10-4=6$; e.g., Campbell \& Xue, 2001; Lefevre et al., 1996). This may pose difficulties for individuals with dyslexia because they have fewer facts available and are less efficient in retrieving them. The latter also puts higher demands on working memory, which might lead to slower performance in the execution of procedural strategies.

Consistent with previous research (Ramus et al., 2003; Snowling, 2000; Vellutino et al., 2004), we showed that adults with dyslexia had weaker phonological processing skills than controls. Together with the observation that these same individuals with dyslexia also retrieved fewer arithmetic facts, these data indicate that weaker phonological processing skills coincide with less fact retrieval from long-term memory. The correlational analyses showed that phonological processing, particularly phonological awareness, was related to fact retrieval. These associations were found in both control and dyslexia subgroups, even when individual differences in reading ability were controlled for.

Against the background of cognitive neuroimaging data (Gelfand \& Bookheimer, 2003; Pugh et al., 2001; Shaywitz et al., 1998), we expected that those measures that put the highest demands on phonological representations would reveal the strongest associations with arithmetic fact retrieval. It may be contended that the phonological awareness tasks were the most sensitive to participants underlying phonological representations (Elbro, 1996). Indeed, phonological awareness measures appear to correlate more strongly than the other phonological components with implicit measures of phonological representations (Boada \& Pennington, 2006) and with categorical speech perception (e.g., Boets, Ghesquière, van Wieringen, \& Wouters, 2007). This might explain why the correlations between phonological processing and fact retrieval were most prominent on the phonological awareness tasks.

Although the administered phonological awareness tasks required a meta-cognitive level of processing (e.g., Boada \& Pennington, 2006), it seems unlikely that some form of metalinguistic awareness in itself is crucial for successful arithmetic fact retrieval. Rather, we contend that in the current study the phonological awareness tasks were the most sensitive to the quality of underlying phonological representations. Our findings suggest that more distinct phonological representations will add to the development of more arithmetic facts and to more efficient arithmetic fact retrieval. Future studies should test this hypothesis more rigorously by using phonological processing tasks that measure phonological representations without meta-linguistic processing requirements, such as a lexical gating task or a syllable similarity task (see Boada \& Pennington, 2006 for a similar rationale in reading research).

It remains possible that other abilities that influence performance on phonological awareness tasks account for the relation between phonological awareness and fact retrieval. Indeed, this relation might have occurred due to the covariation of both abilities with reading skill. However, our findings showed that the associations between phonological awareness and multiplication fact retrieval remained when reading ability was additionally controlled for, which renders this explanation unlikely.

It is important to note that the subgroup analyses showed that the association between phonological processing and fact retrieval was more prominent in multiplication than in subtraction. This 
is in line with the triple-code model, which postulates that multiplication but not subtraction relies on a verbal code (Dehaene, 1992; Dehaene \& Cohen, 1995; Dehaene et al., 2003). It fits with neuroimaging findings that show more activation in the left angular gyrus for multiplication as compared to subtraction (Chochon et al., 1999; Lee, 2000). These findings are also in accordance with patient studies: Temple (1991) reported a patient with reading deficits who had impaired multiplication but spared subtraction; in patients with acalculia, lesions that affect the left perisylvian language areas but spare the intraparietal sulcus typically show deficits in multiplication but not in subtraction (see Dehaene et al., 2003 for a review). This all indicates that multiplication rather than subtraction is dependent on the phonological system.

Why were the associations between phonological processing and fact retrieval more prominent in multiplication than in subtraction? While multiplication is often learned by rote memorization, subtraction is usually taught as the inverse operation of addition. Subtraction problems might then be solved by retrieving their corresponding addition fact (e.g., $8-5=$ might be solved by knowing that $5+3=8$ ), a process that is not merely (asemantic) rote-learned but requires some form of quantity manipulation. This might explain why no significant associations between phonological processing and subtraction fact retrieval were observed at the subgroup level.

\section{Conclusion}

The triple-code model, cognitive neuroimaging and developmental data point to a specific relation between phonological processing and arithmetic fact retrieval. As a result, individuals with deficits in phonological processing, such as individuals with developmental dyslexia, are expected to show poorer arithmetic fact retrieval. Although this has been suggested theoretically, little systematic empirical evidence exists.

The present study showed that individuals with developmental dyslexia without dyscalculia indeed retrieved fewer arithmetic facts from memory and were less efficient in doing so. At the same time, these individuals had a deficit in phonological processing. Phonological processing, particularly phonological awareness, was related to arithmetic fact retrieval. This association was especially prominent in multiplication, indicating that fact retrieval in multiplication rather than subtraction is mediated by phonological processes.

Our findings have important implications for future neuroimaging studies. Although the present investigation was guided by cognitive neuroscience research, none of the reported imaging studies examined phonological processing and fact retrieval in the same sample. Our data point to a behavioral association between multiplication fact retrieval and phonological processing. Future neuroimaging studies should examine this at the neural level, expecting similar activation patterns in the left temporo-parietal areas during a phonological awareness task and during the retrieval of multiplication facts.

\section{Acknowledgements}

We wish to thank all individuals who participated in this study. Bert De Smedt and Bart Boets are Postdoctoral Fellows of the Research Foundation Flanders, Belgium. This research was partially supported by grants G0216.02 and G0331.08 of the Research Foundation Flanders (FWO), Belgium, and by a grant of the Research Council of Katholieke Universiteit Leuven, OT/07/034. Special thanks are due to Maaike Vandermosten and Hanne Poelmans for their assistance with the selection and testing of the participants. We thank Pol Ghesquière and Jan Wouters for their valuable comments on this study.

\section{References}

Boada, R., \& Pennington, B. F. (2006). Deficient implicit phonological representations in children with dyslexia. Journal of Experimental Child Psychology, 95, 153-193.

Boets, B., Ghesquière, P., van Wieringen, A., \& Wouters, J. (2007). Speech perception in preschoolers at family risk for dyslexia: Relations with low-level auditory processing and phonological ability. Brain and Language, 101, 19-30.

Bowers, P. G., \& Ishaik, G. (2003). RAN's contribution to understanding reading disabilities. In H. L. Swanson, K. R. Harris, \& S. Graham (Eds.), Handbook of learning disabilities (pp. 140-157). New York, NY: The Guilford Press.

Butterworth, B. (2005). Developmental dyscalculia. In J. I. D. Campbell (Ed.), Handbook of mathematical cognition (pp. 455-467). Hove, UK: Psychology Press.

Campbell, J. I. D., \& Xue, Q. L. (2001). Cognitive arithmetic across cultures. Journal of Experimental Psychology General, 130, 299-315.

Chochon, F., Cohen, L., Van De Moortele, P. F., \& Dehaene, S. (1999). Differential contributions of the left and right inferior parietal lobules to number processing. Journal of Cognitive Neuroscience, 11, 617-630.

Dehaene, S. (1992). Varieties of numerical abilities. Cognition, 44, 1-42.

Dehaene, S., \& Cohen, L. (1995). Towards an anatomical and functional model of number processing. Mathematical Cognition, 1, 83-120.

Dehaene, S., Piazza, M., Pinel, P., \& Cohen, L. (2003). Three parietal circuits for number processing. Cognitive Neuropsychology, 20, 487-506.

De Rammelaere, S., Stuyven, E., \& Vandierendonck, A. (1999). The contribution of working memory resources in the verification of simple mental arithmetic sums. Psychological Research-Psychologische Forschung, 62, 72-77.

De Smedt, B., Taylor, J., Archibald, L., \& Ansari, D. (2010). How is phonological processing related to individual differences in children's arithmetic skills. Developmental Science, 13, 508-520.

Elbro, C. (1996). Early linguistic abilities and reading development: A review and a hypothesis. Reading and Writing, 8, 453-485.

Fuchs, L. S., Compton, D. L., Fuchs, D., Paulsen, K., Bryant, J. D., \& Hamlett, C. L. (2005). The prevention, identification, and cognitive determinants of math difficulty. Journal of Educational Psychology, 97, 493-513.

Gathercole, S. E, Willis, C. S., Baddeley, A. D., \& Emslie, H. (1994). The Children's Test of Nonword Repetition: A test of phonological working memory. Memory, 2, 103-127.

Gelfand, J. R., \& Bookheimer, S. Y. (2003). Dissociating neural mechanisms of temporal sequencing and processing phonemes. Neuron, 38, 831-842.

Gersons-Wolfensberger, D. C. M., \& Ruijssenaars, A. J. J. M. (1997). Definition and treatment of dyslexia: A report by the Committee on Dyslexia of the Health Council of the Netherlands. Journal of Learning Disabilities, 30, 209-213.

Göbel, S. M., \& Snowling, M. J. (2010). Number processing skills in adults with dyslexia. The Quarterly Journal of Experimental Psychology, 63, 1361-1373.

Grabner, R. H., Ansari, D., Koschutnig, K., Reishofer, G., Ebner, F., \& Neuper, C. (2009). To retrieve or to calculate? Left angular gyrus mediates the retrieval of arithmetic facts during problem solving. Neuropsychologia, 47, 604-608.

Grabner, R. H., Ansari, D., Reishofer, G., Stern, E., Ebner, F., \& Neuper, C. (2007). Individual differences in mathematical competence predict parietal brain activation during mental calculation. Neuroimage, 38, 346-356.

Hecht, S. A., Torgesen, J. K., Wagner, R. K., \& Rashotte, C. A. (2001). The relations between phonological processing abilities and emerging individual differences in mathematical computation skills: A longitudinal study from second to fifth grades. Journal of Experimental Child Psychology, 79, 192-227.

Holloway, I. D., \& Ansari, D. (2009). Mapping numerical magnitudes onto symbols: The numerical distance effect and individual differences in children's mathematics achievement. Journal of Experimental Child Psychology, 103, 17-29.

Landerl, K., Bevan, A., \& Butterworth, B. (2004). Developmental dyscalculia and basic numerical capacities: A study of 8-9-year-old students. Cognition, 93, 99-125.

Landerl, K., \& Wimmer, H. (2000). Deficits in phoneme segmentation are not the core problem of dyslexia: Evidence from German and English children. Applied Psycholinguistics, 21, 246-262.

Lee, K. M. (2000). Cortical areas differentially involved in multiplication and subtraction: A functional magnetic resonance imaging study and correlation with a case of selective acalculia. Annals of Neurology, 48, 657-661.

Lee, K. M., \& Kang, S. Y. (2002). Arithmetic operation and working memory: Differential suppression in dual tasks. Cognition, 83, B63-B68.

Lefevre, J., Sadesky, G. S., \& Bisanz, J. (1996). Selection of procedures in mental addition: Reassessing the problem size effect in adults. Journal of Experimental Psychology-Learning Memory and Cognition, 22, 216-230.

McCandliss, B. D., \& Noble, K. G. (2003). The development of reading impairment: A cognitive neuroscience model. Mental Retardation and Developmental Disabilities Research Reviews, 9, 196-205.

Miles, T. R. (1983). Dyslexia: The pattern of difficulties. Springfield, IL: Charles C Thomas.

Moyer, R. S., \& Landauer, T. K. (1967). Time required for judgements of numerical equality. Nature, 215, 1519-1520.

Nicolson, R. I., \& Fawcett, A. J. (1994). Reaction times and dyslexia. Quarterly Journal of Experimental Psychology A: Human Experimental Psychology, 47, 29-48.

Piazza, M., Izard, V., Pinel, P., Le Bihan, D., \& Dehaene, S. (2004). Tuning curves for approximate numerosity in the human intraparietal sulcus. Neuron, 44 , 547-555.

Pugh, K. R., Mencl, W. E., Jenner, A. R., Katz, L., Frost, S. J., Lee, J. R., et al. (2001). Neurobiological studies of reading and reading disability. Journal of Communication Disorders, 34, 479-492.

Ramus, F. (2003). Developmental dyslexia: Specific phonological deficit or general sensorimotor dysfunction? Current Opinion in Neurobiology, 13, 212-218. 
Ramus, F., Rosen, S., Dakin, S. C., Day, B. L., Castellote, J. M., White, S., et al. (2003). Theories of developmental dyslexia: Insights from a multiple case study of dyslexic adults. Brain, 126, 841-865.

Scheltinga, F. (2003). The Dutch Nonword Repetition Test. Unpublished manuscript, University of Amsterdam, The Netherlands.

Schlaggar, B. L., \& McCandliss, B. D. (2007). Development of neural systems for reading. Annual Review of Neuroscience, 30, 475-503.

Schneider, W., Eschmann, A., \& Zuccolotto, A. (2002). E-prime reference guide. Pittsburgh, PA: Psychology Software Tools.

Seitz, K., \& Schumann Hengsteler, R. (2000). Mental multiplication and working memory. European Journal of Cognitive Psychology, 12, 552-570.

Shalev, R. (2007). Prevalence of developmental dyscalculia. In D. B. Berch, \& M. M. M. Mazzocco (Eds.), Why is math so hard for some children? The nature and origins of mathematical learning difficulties and disabilities (pp. 49-60). Baltimore, MA: Paul H Brookes Publishing.

Shaywitz, S. E., Shaywitz, B. A., Pugh, K. R., Fulbright, R. K., Constable, R. T., Mencl, W. E., et al. (1998). Functional disruption in the organization of the brain for reading in dyslexia. Proceedings of the National Academy of Sciences of the United States of America, 95, 2636-2641.

Simmons, F. R., \& Singleton, C. (2006). The mental and written arithmetic abilities of adults with dyslexia. Dyslexia, 12, 96-114.

Simmons, F. R., \& Singleton, C. (2008). Do weak phonological representations impact on arithmetic development? A review of research into arithmetic and dyslexia. Dyslexia, 14, 77-94.

Snowling, M. J. (2000). Dyslexia (2nd ed.). Malden, MA: Blackwell Publishers.

Temple, C. M. (1991). Procedural dyscalculia and number fact dyscalculia: Double dissociation in developmental dyscalculia. Cognitive Neuropsychology, 8, 155-176.
Temple, E. (2002). Brain mechanisms in normal and dyslexic readers. Current Opinion in Neurobiology, 12, 178-183.

Turner Ellis, S. A., Miles, T. R., \& Wheeler, T. J. (1996). Speed of multiplication in dyslexics and non-dyslexics. Dyslexia, 2, 121-139.

van Daal, V., \& van der Leij, A. (1999). Developmental dyslexia: Related to specific or general deficits? Annals of Dyslexia, 49, 71-104.

van den Bos, K. P., Spelberg, H. C. L., Scheepstra, A. J. M., \& De Vries, J. R. (1994). De Klepel. Vorm A en B. Een test voor de leesvaardigheid van pseudowoorden [Standardized test of reading ability Form A and B]. Nijmegen: Berkhout.

van den Bos, K. P., Zijlstra, B. J. H., \& Spelberg, H. C. (2002). Life-span data on continuous-naming speeds of numbers, letters, colors, and pictured objects, and word-reading speed. Scientific Studies of Reading, 6, 25-49.

Vellutino, F. R., Fletcher, J. M., Snowling, M. J., \& Scanlon, D. M.(2004). Specific reading disability (dyslexia): What have we learned in the past four decades? Journal of Child Psychology and Psychiatry, 45(1), 2-40.

Vigneau, M., Beaucousin, V., Hervé, P. Y., Duffau, H., Crivello, F., Houdé, O., et al. (2006). Meta-analyzing left hemisphere language areas: Phonology, semantics and sentence processing. NeuroImage, 30, 1414-1432.

Wagner, R. K., \& Torgesen, J. K. (1987). The nature of phonological processing and its causal role in the acquisition of reading skills. Psychological Bulletin, 101, $192-212$.

Willburger, E., Fussenegger, B., Moll, K., Wood, G., \& Landerl, K. (2008). Naming speed in dyslexia and dyscalculia. Learning and Individual Differences, 18, 224236.

Wilson, A. J., \& Dehaene, S. (2007). Number sense and developmental dyscalculia. In D. Coch, G. Dawson, \& K. Fischer (Eds.), Human behavior, learning and the developing brain: Atypical development (pp. 212-238). New York, NY: Guilford Press. 